\title{
ESTUDO DE BIOMASSA DE Tectona grandis L.f. SOB DIFERENTES ESPAÇAMENTOS NO ESTADO DE MATO GROSSO ${ }^{1}$
}

\author{
Eliazel Vieira Rondon²
}

\begin{abstract}
RESUMO - O presente trabalho, conduzido no Município de Sinop, Estado de Mato Grosso, teve por objetivo avaliar a circunferência, a altura total e a produção de biomassa de plantas de Tectona grandis, em diferentes densidades populacionais, obtidas com diferentes espaçamentos e arranjos de plantio. Foram avaliados os espaçamentos de $3 \times 2 \mathrm{~m}, 3 \times 3 \mathrm{~m}, 4 \times 3 \mathrm{~m}, 4 \times 4 \mathrm{~m}, 5 \times 3 \mathrm{~m}, 5 \times 4 \mathrm{~m}$ e $5 \times 5 \mathrm{~m}$, com densidades populacionais que variaram de 400 a 1.666 plantas por hectare. Selecionaram-se três árvores por tratamento, constituindo a individualização dos componentes folhas, galhos e tronco. O experimento teve duração de 76 meses. O aumento da densidade populacional promoveu diminuição na circunferência das plantas de teca, enquanto a altura permaneceu constante. No espaçamento mais denso, a produção de biomassa total da parte aérea teve aumento nas plantas de tectona grandis, concentrando-se principalmente no tronco da árvore.
\end{abstract}

Palavras-chave: Teca, densidade populacional, produção de biomassa e espaçamento.

\section{BIOMASS STUDY OF Tectona grandis L.f. UNDER DIFFERENT SPACING CONDITIONS IN THE NORTH OF MATO GROSSO STATE}

\begin{abstract}
This work was carried out in the municipality of Sinop, State of Mato Grosso with the objective of evaluating the circumference, total height and biomass production of Tectona grandis plants, in different population densities, obtained with different spacings and planting arrangements. The spacings $3 \times 2 \mathrm{~m}, 3 \times 3$ $m, 4 \times 3 m, 4 \times 4 m, 5 \times 3 m, 5 \times 4 m$ and $5 \times 5 m$, were evaluated with the population density varying from 400 to 1.666 plants / hectare. Three trees were selected per treatment, with the individualization consisted of leaves, branches and trunk components. The experiment lasted for 76 months. The increase in population density caused a decrease in teak tree circumference, whereas the height was constant. There was increase in total biomass production of the aerial part of the Tectona grandis plants in the denser spacings, concentrated mainly in the tree trunk.
\end{abstract}

Keywords: Tectona grandis, population density, biomass production and spacing.

\section{INTRODUÇÃO}

A teca (Tectona grandis) é uma espécie arbórea da família Verbenaceae. A área de ocorrência natural dessa espécie situa-se entre $10^{\circ}$ e $25^{\circ} \mathrm{N}$ no subcontinente índico e no sudeste asiático, especialmente na Índia, Burma, Tailândia, Laos, Camboja, Vietnã e Java. Produz uma das madeiras mais belas e melhores que existem, com excelente qualidade em todos os aspectos, podendo ser utilizada para as mais diversas finalidades. É considerada como insuperável na construção naval, sendo adequada para todo o tipo de construções dentro e fora de água, bem como para interiores luxuosos e mobiliário de alto preço (LAMPRECHT, 1990).

No Brasil, os plantios de teca iniciaram-se no final da década de 1960, implantados pela empresa Cáceres Florestal S.A., na região do Município de Cáceres Grosso

\footnotetext{
${ }^{1}$ Recebido em 30.08.2004 e aceito para publicação em 05.04.2006.

${ }^{2}$ EMPAER-MT, Rua das pitangueiras, 84, 78550-00 Sinop-MT, Tel. (66) 531-1215 e Fax. (66) 531-1611.

E-mail: <rondonev@ hotmail.com>.
} 
(TSUKAMOTO FILHO et al., 2003). Os trabalhos relacionados com a silvicultura dessa espécie ainda são poucos.

A definição do espaçamento para o plantio de espécies florestais é de grande importância, uma vez que pode afetar o crescimento das plantas, a exploração florestal e os custos de produção (DANIEL et al., 1982; BALLONI, 1983; CONTRERAS-MARQUES, 1997), possibilitando a otimização dos recursos luz, água e nutriente (REIS e REIS, 1993).

O espaçamento utilizado atualmente, pelas principais empresas reflorestadoras do Brasil, tem sido escolhido visando possibilitar a mecanização das atividades de implantação, manutenção e exploração dos maciços florestais, motivo pelo qual tem sido dado preferência aos espaçamentos com aproximadamente $3 \mathrm{~m}$ entre linhas (BERNARDO, 1995). Entretanto, o espaçamento pode afetar o desenvolvimento e a produtividade das florestas plantadas, principalmente para as espécies de rápido crescimento. Espaçamento inadequado pode acentuar os efeitos da deficiência hídrica sobre as plantas, diminuindo a produtividade da floresta, em razão da intensa competição intra-específica por água, nutrientes, luz e espaço (LELES et al., 1998).

Conforme Patiño-Valera (1986), o espaçamento ótimo é aquele capaz de produzir o maior volume de produto em tamanho, forma e qualidade desejáveis, sendo função da espécie do sítio e do potencial genético do material reprodutivo que for utilizado.

Vale et al. (1982) e Leite et al. (1997) observaram que a adoção de espaçamentos muito reduzidos acarreta a produção de toras de pequeno diâmetro e muitas árvores dominadas, o que compromete o volume final produzido.

O presente trabalho teve por objetivo avaliar o crescimento e a produção de biomassa da parte aérea de Tectona grandis L.f., cultivado sob diferentes densidades populacionais, de modo a gerar subsídios para definição do espaçamento mais apropriado para essa espécie, na região norte do Estado de Mato Grosso.

\section{MATERIAL E MÉTODOS}

O estudo foi conduzido na área da Empresa MatoGrossense de Pesquisa, Assistência e Extensão Rural S/A - EMPAER-MT, situada no Município de Sinop, no norte do Estado, com latitude de $11^{\circ} 50$ ' S, longitude $55^{\circ} 38^{\prime} \mathrm{W}$ e altitude de $384 \mathrm{~m}$. As médias das temperaturas máxima e mínima mensal para a região são de 34 e 24 ${ }^{\circ} \mathrm{C}$, respectivamente, com precipitação média anual de $1.922 \mathrm{~mm}$. Essa região é caracterizada como zona de transição edafoclimática entre cerrado e floresta amazônica, e sua vegetação é classificada como floresta semidecidual submontana dossel emergente. Seu clima, segundo KÖEPPEN, é do tipo Aw (BRASIL, 1980).

Os solos dessa região são geralmente ácidos, com moderada concentração de alumínio trocável, apresentando baixa capacidade de troca catiônica, e são deficientes em cálcio e fósforo, havendo predominância de latossolos profundos bem drenados e planos.

No Quadro 1 estão apresentadas as características químicas do solo do povoamento de Tectona grandis L. f.

A área foi aberta com trator de esteira, tendo seu material vegetal sido queimado na leira. Noventa dias antes do plantio foram distribuídas cinco toneladas de calcário. A adubação de plantio constou de $100 \mathrm{~g}$ de sulfato de amônia, $200 \mathrm{~g}$ de superfosfato simples e $100 \mathrm{~g}$ de cloreto de potássio por cova, adicionandose também 2 L de cinza, 2 L de esterco de gado curtido e $1 \mathrm{~L}$ de esterco de galinha. Procedeu-se a duas adubações químicas anuais em cobertura (início e fim do período chuvoso), aplicando-se em $2 / 3$ da projeção da copa em sulco, no sistema de meia-lua, na mesma proporção usada para a adubação da cova. Utilizaram-se mudas tipo toco ("stump") de raiz nua, com tamanho médio de $17 \mathrm{~cm}$ de comprimento. O plantio ocorreu em 15 de novembro de 1997 e a avaliação da biomassa do experimento ocorreu em 15 de março de 2004, sendo que nesse período não foi efetuado nenhum desbaste. Foram avaliados os seguintes espaçamentos: $3 \times 2 \mathrm{~m}$, $3 \times 3 \mathrm{~m}, 4 \times 3 \mathrm{~m}, 4 \times 4 \mathrm{~m}, 5 \times 3 \mathrm{~m}, 5 \times 4 \mathrm{~m}$ e $5 \times 5 \mathrm{~m}$. O delineamento utilizado foi em blocos ao acaso, com sete tratamentos (espaçamentos) e três repetições. Foram plantadas 525 plantas no total, tendo sido avaliadas 27 árvores centrais por tratamentos.

As avaliações relativas à altura das plantas e circunferência à altura do peito (CAP) foram realizadas anualmente. Para avaliação da biomassa vegetal da parte aérea, em cada tratamento foram abatidas três árvores com CAP médio (uma por repetição). À medida que foram abatidas as árvores, os galhos eram separados do tronco e as folhas dos galhos foram todas coletadas. 
Quadro 1 - Características químicas do solo do povoamento de Tectona grandis plantado em Sinop

Table 1 -Chemical characteristics of the soil under a Tectona grandis stand in the municipality of Sinop, MT

\begin{tabular}{|c|c|c|c|c|c|}
\hline $\begin{array}{l}\text { Profundidade } \\
\quad(\mathrm{cm})\end{array}$ & $\begin{array}{c}\text { Argila } \\
\%\end{array}$ & $\begin{array}{c}\mathrm{pH} \\
\mathrm{H}_{2} \mathrm{O}\end{array}$ & $\begin{array}{c}\mathrm{MO} \\
\mathrm{dag} / \mathrm{kg}\end{array}$ & $\begin{array}{c}\text { P disponível } \\
\text { mg.dm }\end{array}$ & \\
\hline $0-20$ & 55 & 5,2 & 3,3 & 1,6 & \\
\hline \multirow[t]{2}{*}{$\begin{array}{l}\text { Profundidade } \\
\text { (cm) }\end{array}$} & $\frac{\mathrm{K}}{\text { disponível }}$ & $\begin{array}{c}\% \\
\text { saturação }\end{array}$ & $\begin{array}{c}\mathrm{Ca} \\
\text { trocável }\end{array}$ & $\begin{array}{c}\mathrm{Mg} \\
\text { trocável }\end{array}$ & $\begin{array}{l}\text { CTC } \\
\text { efetiva }\end{array}$ \\
\hline & mg.dm ${ }^{-3}$ & de bases & & $\mathrm{cmol}_{\mathrm{c}} \cdot \mathrm{dm}^{-3}$ & \\
\hline $0-20$ & 11 & 23,3 & 1,0 & 0,6 & 1,88 \\
\hline
\end{tabular}

Todos os componentes tiveram sua massa fresca determinada mediante o uso de uma balança e, em seguida, extraiu-se uma amostra para nova pesagem. Para amostragem do tronco, retiraram-se três discos (base, meio e topo) de $5 \mathrm{~cm}$ de espessura. As amostras foram levadas ao laboratório para secagem em estufa de ventilação forçada a $70^{\circ} \mathrm{C}$ até atingir peso constante, obtendo-se o peso de matéria seca. A partir da relação entre o peso de matéria seca e da matéria fresca, determinou-se o teor de umidade e, finalmente, obtevese a biomassa de cada componente. No entanto, para os três discos do tronco usou-se o teor médio, por isso o peso da matéria seca na parte aérea foi em toneladas por hectare, admitindo-se uma sobrevivência igual a $100 \%$ para todos os tratamentos.

\section{RESULTADOS E DISCUSSÃO}

No Quadro 2 estão apresentados os resultados das análises de variâncias para altura, circunferência à altura do peito (CAP) e biomassa total da parte aérea e de seus componentes obtida para Tectona grandis, em função dos diferentes espaçamentos, aos 76 meses de idade.

Nota-se que não houve diferenças estatísticas para altura da planta. No tocante à circunferência, somente o menor espaçamento $(3 \times 2 \mathrm{~m})$ promoveu redução significativa, tendo sido encontradas maiores CAP, nos espaçamentos $4 \times 3 \mathrm{~m}, 4 \times 4 \mathrm{~m}, 5 \times 3 \mathrm{~m}$ e $5 \times 5 \mathrm{~m}$. Leles et al. (1998) também não verificaram influência do espaçamento no crescimento em altura de plantas de Eucalptus camaldulensis e Eucalptus pellita.

De acordo com Paiva et al. (2001), o espaçamento não afeta o crescimento em altura das plantas. Para esses autores, o melhor espaçamento é aquele que produz o máximo de madeira, em tamanho, forma e qualidade, como o menor custo. A floresta plantada em espaçamento reduzido exige desbaste ou corte em idade muito jovem (três a quarto anos), pois há uma estagnação do crescimento, provocando grande exportação de nutriente, o que não é interessante quando se pensa em sustentabilidade do solo.

No estudo realizado com teca por Pauletto e Passos (2004), no Município de Cáceres,MT, eles verificaram que os espaçamentos $4 \times 2 \times 2 \mathrm{~m}$ (fileira duplas), $5 \times$ $2 \mathrm{~m}$ e $6 \times 2 \mathrm{~m}$ promoveram os melhores DAP e volume aos 41 meses de idade.

Rondon (2002), trabalhando com sete espaçamentos para Schizolobium amazonicum, no norte de Mato Grosso, concluiu que os espaçamentos 4 x $3 \mathrm{~m} \mathrm{e} 4$ x $4 \mathrm{~m}$ proporcionaram maiores crescimentos, enquanto a produção de biomassa total foi superior nos espaçamentos $4 \times 4 \mathrm{~m} \mathrm{e} 4 \times 2 \mathrm{~m}$.

Quanto à produção de biomassa total da parte aérea das plantas, bem como seus componentes, percebeu-se inicialmente que o espaçamento testado conduziu a diferentes produções de biomassa total. Verificou-se que a maior contribuição foi do tronco com $74,57 \%$, seguindo dos galhos $(18,52 \%)$ e folhas $(6,91 \%)$ da produção de biomassa total da biomassa (Figura 1). Observou-se em povoamento de E. grandis, com idades de sete anos, que o tronco contribuiu com $94,8 \%$ de toda a matéria seca (BELLOTE, 1979). As menores produções para o total da biomassa estão relacionadas aos espaçamentos $5 \times 5 \mathrm{~m}, 5 \times 4 \mathrm{~m}$ e 5 x $3 \mathrm{~m}$ e as maiores, aos espaçamentos $3 \times 2 \mathrm{~m}, 3 \times 3$ m e 4 × $3 \mathrm{~m}$, sendo que o espaçamento de $3 \times 2 \mathrm{~m}$ possibilitou o maior acúmulo de biomassa total. Dados semelhantes foram também observados com Eucalyptus spp por Pereira (1990), Bernardo (1995) e Leles (1995).

A influência de diferentes espaçamentos sobre a produção de biomassa varia com a espécie, a idade das plantas e a qualidade de sítio. Nos espaçamentos mais densos, a maior competição entre plantas resulta na estabilização do acúmulo de biomassa em menor idade que aquela observada em espaçamentos mais amplos (BERNARDO, 1995).

Levando em consideração os resultados de altura e biomassa, verificou-se a necessidade de estudos de árvores em idade mais avançada, de maneira que se possa inferir sobre a época em que ocorrerá o equilíbrio na produção de biomassa da madeira nos diferentes espaçamentos para as condições de sítio em estudo.

R. Árvore, Viçosa-MG, v.30, n.3, p.337-341, 2006 
Quadro 2 - Comparação das médias para altura, circunferência à altura do peito (CAP), matéria seca das folhas, dos galhos, do tronco e total de Tectona grandis em diferentes espaçamentos, aos 76 meses de idade

Table 2 - Mean comparison of breast height circumference, height, total dry matter and dry matter of leaves, branches and trunk of Tectona grandis, in different spacings at 76 months of age

\begin{tabular}{|c|c|c|c|c|c|c|}
\hline Espaçamento & Altura & CAP & Folhas & Galhos & Tronco & Total \\
\hline$(\mathrm{m})$ & $(\mathrm{m})$ & $(\mathrm{cm})$ & \multicolumn{4}{|c|}{$\mathrm{t} / \mathrm{ha}^{-1}$} \\
\hline $3 \times 2$ & $9,0 \mathrm{~A}$ & $36,31 \mathrm{~B}$ & $4,49 \mathrm{~A}$ & $12,49 \mathrm{~A}$ & $41,38 \mathrm{~A}$ & $58,36 \mathrm{~A}$ \\
\hline $3 \times 3$ & $11,0 \mathrm{~A}$ & $42,91 \mathrm{~A}$ & $4,99 \mathrm{~A}$ & $10,77 \mathrm{~A}$ & $38,69 \mathrm{~A}$ & $54,45 \mathrm{~B}$ \\
\hline $4 \times 3$ & $11,0 \mathrm{~A}$ & $44,20 \mathrm{~A}$ & $2,99 \mathrm{~A}$ & $8,49 \mathrm{AB}$ & $33,73 \mathrm{~A}$ & $45,21 \mathrm{C}$ \\
\hline $4 \times 4$ & $9,0 \mathrm{~A}$ & $45,64 \mathrm{~A}$ & $1,87 \mathrm{BC}$ & $5,37 \mathrm{~B}$ & $25,98 \mathrm{~B}$ & $33,22 \mathrm{D}$ \\
\hline $5 \times 3$ & $9,0 \mathrm{~A}$ & $43,44 \mathrm{~A}$ & $1,99 \mathrm{C}$ & $5,92 \mathrm{~B}$ & $23,55 \mathrm{~B}$ & $31,46 \mathrm{D}$ \\
\hline $5 \times 4$ & $10,0 \mathrm{~A}$ & $41,75 \mathrm{~A}$ & $1,20 \mathrm{CD}$ & $3,90 \mathrm{~B}$ & $17,55 \mathrm{BC}$ & $22,65 \mathrm{E}$ \\
\hline $5 \times 5$ & $10,0 \mathrm{~A}$ & $43,60 \mathrm{~A}$ & $0,40 \mathrm{D}$ & $1,08 \mathrm{BC}$ & $12,52 \mathrm{C}$ & $14,00 \mathrm{~F}$ \\
\hline
\end{tabular}

Médias seguidas pela mesma letra nas colunas não diferem, estaticamente, pelo teste de Tukey a $5 \%$.

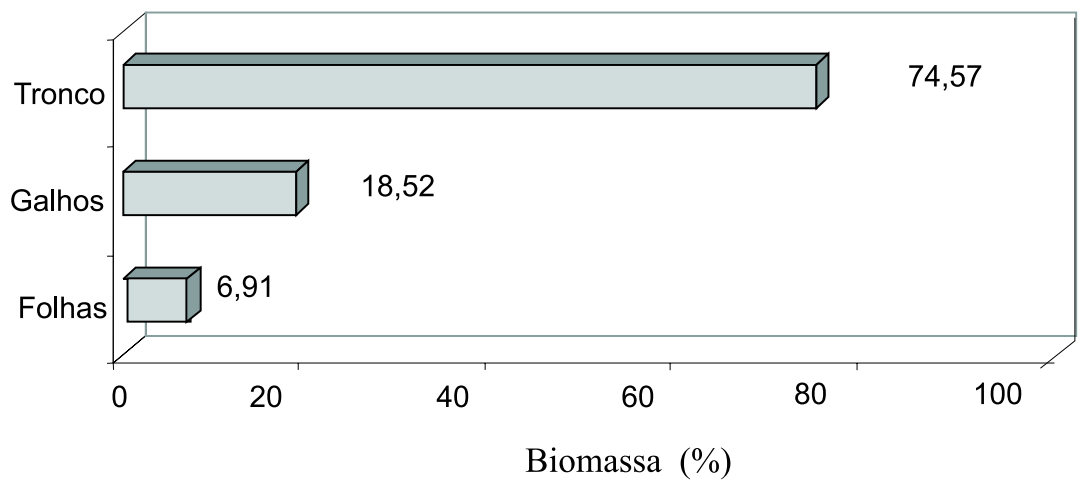

Figura 1 - Distribuição porcentual dos componentes das árvores.

Figure 1 - Percentage distribution of tree components.

Vale ressaltar que a adoção de espaçamentos reduzidos eleva o custo no plantio, tanto na produção de maior número de mudas quanto nas práticas silviculturais. O experimento foi implantado em solo argiloso e de baixa fertilidade. Em contrapartida, a teca é uma espécie que requer solo de alta fertilidade e, com isso, eleva ainda mais os custos de plantio, e a escolha inadequada do espaçamento pode inviabilizar o plantio.

\section{CONCLUSÕES}

- Variações de espaçamento, dentre os estudados, não afetam o crescimento em altura de Tectona grandis.

- Espaçamentos muito reduzidos, como o de $3 \times 2 \mathrm{~m}$, promovem redução na circunferência das árvores.

- Espaçamentos maiores aumentam a circunferência das árvores, porém promovem redução na produção de biomassa do estande.

R. Árvore, Viçosa-MG, v.30, n.3, p.337-341, 2006

\section{REFERÊNCIAS BIBLIOGRÁFICAS}

BALLONI, E. A. Influência do espaçamento de plantio na produtividade florestal. Silvicultura, v. 8, n. 31, p. 558-592, 1983.

BALLONI, E. A.; SIMÕES, J. W. O

espaçamento do plantio e suas implicações Silviculturais. Piracicaba: IPEF, 1980. 16p. (Série Técnica, 3).

BELlote, A. F. J. Concentração, acúmulo e exportação de nutrientes pelo Eucalyptus grandis em função da idade. 1979. 129f. Dissertação (Mestrado em Engenharia Florestal) - Escola Superior de Agricultura Luiz de Queiroz, Piracicaba, 1979. 
BERNARDO, A. L. Crescimento e Eficiência nutricional de Eucapyptus spp. sob diferentes espaçamentos na região de cerrado de Minas Gerais. 1995. 102f. Dissertação (Mestrado em Ciência Florestal) - Universidade Federal de Viçosa, Viçosa, 1995.

BRASIL. Ministério de Minas de Energia: Departamento Nacional da produção mineral. Projeto RADAM Brasil. Folhas sc. 21. Juremo: Geomorfologia, pedologias, vegetação e uso potencial da terra. Rio de Janeiro: 1980. 460p. (Levantamento de recursos naturais, v. 20).

CONTRERAS-MARQUEZ, C.E. Estudo silvicultural e econômico de povoamentos e eucalipto na região de cerrado de Minas Gerais. 1997. $131 \mathrm{f}$. Dissertação (Mestrado em Ciência Florestal)Universidade Federal de Viçosa, Viçosa, 1997.

DANIEL, T. W.; HELMS, J. A.; BACKER, F. S. Princípios de silvicultura. México: McGraw-Hill, 1982. 492p.

LAMPRECHT, H. Silvicultura nos trópicos: ecossistemas florestais e respectivas espécies arbóreas- possibilidades e métodos de povoamento sustentado. Eschborn: Instituto de Silvicultura da Universidade de Göttingen, 1990. p.310-313.

LEITE, F. P. et al. Crescimento de Eucalyptus grandis em diferentes densidades populacionais. Revista Árvore, v. 21, n. 3, p. 313-321, 1997.

LELES, P. S. S. Crescimento alocação de biomassa e distribuição de nutrientes e uso de água em $\boldsymbol{E}$. camaldulensis e $\boldsymbol{E}$. pellita sob diferentes espaçamentos. 1995. 133f. Dissertação (Mestrado em Solo e Nutrição de Plantas) Universidade Federal de Viçosa, Viçosa, 1995.

LELES, P. S. S. et al. Relações hídricas e crescimento de árvores de Eucalyptus canaldulensis e Eucalyptus pellita sob diferentes espaçamentos na região de cerrado. Revista Árvore, v. 22, n. 1, p. 41-50, 1998.
PATIÑO-VALERA, F. Variação genética em Progênies de Eucalyptus saligna Smith e sua interação com espaçamento. 1986. 192f. Dissertação (Mestrado em Engenharia Florestal) - Escola Superior de Agricultura "Luiz de Queiroz", Piracicaba, 1986.

PAIVA, H. N.; JACOVINE, L. A, G.; REBEIRO, G. T. Cultivo de eucalipto em propriedade rurais. Viçosa - MG: Aprenda Fácil, 2001. p. 66-67.

PAULETTO, D.; PASSOS, C.A.M. Influência do espaçamento e solo no crescimento de Tectona grandis, L.F. no município de Cáceres- MT. In: CONGRESSOEEXPOSIÇÃOINTERNACIONAL SOBREFLORESTAS-REDESCOBRINDOO CERRADO, 7., 2004, Brasília. Resumos Técnicos... Brasília: BIOSFERA, 2004. P.87-88.

PEREIRA, A. R. Biomassa e ciclagem de nutrientes minerais em povoamentos jovens de Eucalyptus grandis e Eucalyptus urophylla em região de cerrado. 1990. 167f. Tese (Doutorado em Solos e Nutrição de Plantas) Universidade Federal de Viçosa, Viçosa, 1990.

REIS, G. G.; REIS, M. G. F. Competição por luz, água e nutrientes em povoamentos florestais. In: SIMPÓSIO BRASILEIRO DE PESQUISA

FLORESTAL,1., 1993, Belo Horizonte.

Resumos... Viçosa: SIF/UFV, 1993. p. 161-172.

RONDON, E. V. Produção de biomassa e crescimento de árvores de Schizolobium amazonicum (Hub.) Ducke sob diferentes espaçamentos na região de mata. Revista Árvore, v. 26 n. 5, p. 573-576, 2002.

SIMÕES, J. W.; BRANDI, T. M.; LEITE, N. B. Formação, manejo e exploração de florestas de rápido crescimento. Brasília: IBDF, 1981. 131p.

TSUKAMOTO FILHO, A. A. et al. Análise econômica de um plantio de teça submetido a desbastes. Revista Árvore, v. 27, n. 4, p. 487-494, 2003.

VALE, A B. et al. Influência do espaçamento e do sítio na produção Florestal. Viçosa, MG: SIF, 1982. 20p. (Boletim Técnico, 4).

R. Árvore, Viçosa-MG, v.30, n.3, p.337-341, 2006 